\title{
Young 4ever $†$-the use of capillarity for passive flow handling in lab on a chip devices
}

DOI: $10.1039 / \mathrm{b} 613839 \mathrm{j}$

\section{Introduction}

When we do our everyday fluid handling in the laboratory such as filling, emptying, metering and cleaning, we often don't realize that we use a set of everyday forces that are 'always there' because they just go without saying. These forces are gravity, suction and the capillary force, for the application of which we need no sophisticated equipment. Apart from their use for fluid handling they were therefore also the first forces to be used for liquid propulsion in separation and analysis systems in the 19th and 20th century laboratory, e.g., the capillary force in thin layer chromatography or in test strips (from $\mathrm{pH}$ paper to clinical test strips) and gravity in liquid chromatography. To these everyday forces for fluid handling we could also add the centrifugal force (e.g., in hand-operated bench top centrifuges) and evaporation.

The ready availability of these everyday forces makes them particularly suited for fluid handling and propulsion in diagnostics and point-of-care (POC) devices. When such systems are miniaturized, the capillary force scales the best because it is exerted at the contact perimeter of liquid and channel wall, and the perimeter becomes larger relative to the channel cross-sectional area on downscaling. The capillarity-induced pressure, which is the capillary force per unit channel area, is therefore inversely proportional to the characteristic channel dimension as shown in Table 1. Classically, paper, fabric, membranes or mesh is used for capillary propulsion and flow control, for example in glucose strips. ${ }^{1}$ Precise flow control and metering however can be problematic in such devices and the past few years have therefore seen a surge in interest in microfluidic POC devices employing capillarity. Also outside the area of

$\uparrow$ A reference to the Young-Laplace equation.
POC devices the interest in the use of capillarity in LOC devices has recently increased. This Focus article attempts to give an overview of this developing field, and will show that there is still a lot of potential in the capillary phenomena described in 1805 by Young and de Laplace.

\section{Capillarity-induced pressure and liquid flow}

The microfluidic devices that employ capillarity often use a local constriction or tapering of the channel to control liquid flow, and in these cases the capillary pressure can best be derived from the total change in system energy on filling. When the channel fills or empties, the solid/liquid interfacial area $A_{\mathrm{sl}}$ and the liquid/gas interfacial area $A_{\mathrm{lg}}$ change with the change of the liquid volume $V_{1}$ inside the channel. The capillary pressure can then be calculated $\mathrm{as}^{2}$

$$
P_{\text {cap }}=-\gamma_{\mathrm{lg}}\left(\cos \theta \frac{\mathrm{d} A_{\mathrm{sl}}}{\mathrm{d} V_{1}}-\frac{\mathrm{d} A_{\mathrm{lg}}}{\mathrm{d} V_{1}}\right),
$$

with $\gamma_{\lg }\left(\mathrm{N} \mathrm{m}^{-1}\right)$ the surface tension of the liquid and $\theta$ the contact angle. Spontaneous imbibition occurs when the capillarity-induced pressure is negative, which is true if the term between the brackets is positive. For wetting liquids $\left(\theta<90^{\circ}\right)$ in widening channels this occurs only when the first term between the brackets, representing the energy gain by wetting the channel walls, is larger than the second bracketed term, representing the energy loss due to the increase of the liquid/gas contact area. At constant cross section eqn (1) can be simplified. For example for a rectangular channel of width $\gg$ height $h, \mathrm{~d} A_{\mathrm{sl}} / \mathrm{d} V_{1}=$ $2 / h$ and $\mathrm{d} A_{1 \mathrm{~g}} / \mathrm{d} V_{1}=0$ so that we obtain the Young-Laplace equation

$$
P_{\text {cap }}=-\gamma_{\text {lg }} \cos \theta\left(\frac{2}{h}\right) \text {. }
$$

Most applications of capillarity in LOC use wetting conditions, which cause spontaneous imbibition. Liquid movement in these systems is truly 'automatic' (self-moving) or autonomous, and after reference $^{3}$ we will here refer to them as autonomous capillary systems (ACS). In a wetting channel of constant cross section and surface properties (constant $P_{\text {cap }}$ ), the meniscus proceeds with the square root of time due to the linear increase of flow resistance with filled channel length, and the time-dependent meniscus position is given by the LucasWashburn equation. ${ }^{4}$ The flow speed does not need to decrease in time however if channel cross sections can be smartly tailored as in some of the cases (as cited below) it is demonstrated that the flow speed can be made constant in time. ${ }^{3}$

\section{Flow control}

Eqn (1) indicates that the capillary pressure can be tuned by changing the contact angle $\theta$ or the device geometry (assuming constant $\gamma$ ). Control can thereby be exerted at the advancing liquid front (upstream) or at the rear of

Table 1 Pressures generated by capillarity, vacuum and gravity in circular channels of different radius. The capillarity-induced pressure scales favourably

\begin{tabular}{llll}
\hline $\begin{array}{l}\text { Channel } \\
\text { radius } / \mu \mathrm{m}\end{array}$ & $\begin{array}{l}\text { Capillarity-induced } \\
\text { pressure } / \text { bar }\end{array}$ & Vacuum $/$ bar & $\begin{array}{l}\text { Gravity-induced } \\
\text { pressure } / \text { bar }\end{array}$ \\
\hline 100 & 0.014 & 1 & 0.001 \\
10 & 0.14 & 1 & 0.001 \\
1 & 1.4 & 1 & 0.001 \\
${ }^{a}$ Surface tension $=$ & $0.07 \mathrm{~N} \mathrm{~m}^{-1}$, contact angle $=0^{\circ} .{ }^{b}$ Reservoir height $1 \mathrm{~cm}$. \\
\hline
\end{tabular}




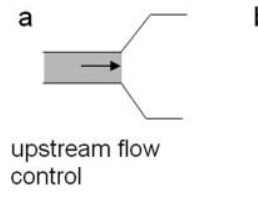

b

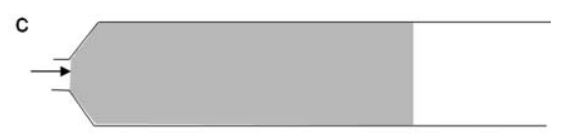

downstream flow control

Fig. 1 Flow control in autonomous capillary systems. (a) A widening of the channel, pinning the meniscus (see eqn (1)); (b) upstream flow control: a Y-junction or liquid AND gate, where the liquid becomes pinned if only one feed channel is filled, but flow proceeds if both channels are filled; (c) downstream flow control: pinning of the liquid rear at a channel constriction (capillary retention valve).

a liquid plug (downstream). Fig. 1 shows an example of both control approaches in ACSs, with an example of upstream flow control in Fig. 1b. At the Y-junction shown, the flow will only proceed when both top channels are filled with liquid, and it can thus be regarded as a fluidic AND gate. ${ }^{9}$ Liquid flow automatically proceeds in the upstream hydrophilic channels until the widening part is reached where the liquid stops as the capillary pressure becomes zero. At this meniscus position the two bracketed terms in eqn (1) cancel and so-called meniscus or contact-line pinning occurs (Fig. 1a). ${ }^{5,6}$ The fluid flow can now only resume once liquid has arrived from the second neighbouring channel, leading to a merging of the menisci and again a negative capillary pressure. Fig. 1c shows an example of downstream flow control in an ACS, namely a downstream narrowed channel section. As soon as the liquid supply from the rear is exhausted, the rear meniscus stops at the point where the local capillary pressure is more negative than at the front meniscus. Liquid flow is now resumed only by renewed delivery of liquid from the rear.

Flow control in hydrophobic channel networks is also possible (e.g., by local narrowing) and was first described by McNeely et al. and later by Ahn et al..$^{7,8}$ A clear disadvantage of hydrophobic networks is, that an external pressure source, suction or actuation must be applied, necessitating additional equipment. Ref. 9 reviews several such actuation methods.

\section{Capillary flow in test strips}

Though 'test strip' might not sound very impressive, these devices form the backbone of POC diagnostics and actually allow quite complicated assays to be performed. In the past 20 years test strips, also called lateral flow assays, have indeed been widely employed for POC assays like pregnancy tests, HIV diagnosis and tests for drugs of abuse. ${ }^{1}$ After introduction of a sample drop on the sample pad of such devices, capillary forces maintain the flow in a microporous matrix transporting and processing the sample. Processes such as filtration, chromatographic separation, dissolution of lines of dried chemicals, diffusion, reaction and accumulation of reaction products thereby occur on the way. ${ }^{10,11}$ Complex tests like sandwich immunoassays are also performed in this format. Flow rates and incubation times can be influenced by tailoring device geometry and local hydrophobicity. This automatic behaviour and easy programmability of capillarity-driven systems is a great advantage and is also used to great advantage in micromachined POC devices, as we will show below.

\section{Capillary flow in LOC POC applications}

The inclusion of microfluidic channels in test strips increases control of the fluidic properties. In 1997 van Damme et al. from Organon Teknica developed POC devices in which they smartly combined what they called the 'fluidic elements' of microfluidic channels, adsorbers and membranes. ${ }^{12}$ Due to the programmability regarding e.g., automatic timing, an ELISA could be successfully implemented. In a more recent development Juncker et al. from IBM Research reported in 2002 the construction of what they called autonomous capillary microfluidic systems (ACS) (Fig. 2). ${ }^{3}$

All liquid transport in the ACS of Juncker et al. is by capillarity-induced pressure. ${ }^{3}$ Instead of relying on adsorber pads (like in test strips) to provide constant and long-lasting suction pressure, the device contains a capillary pump section providing a constant suction pressure. Since the main fluidic resistance resides in two narrow constrictions around the reaction chamber, a constant flow rate is generated in the latter. The device thus presents a fluidic analogue of an electrical current source. Directly at the service port a capillary retention valve (Fig. 1c) gives downstream flow control. Subsequent deliveries of fluid quantities to the service port allow complex assays like sandwich fluorescence immunoassays to be performed in this device. ${ }^{13}$ In the latter case evaporation was employed as an additional driving force to maintain a low volume fluid flow rate for longer periods. The accurate patterning of arrays of different reagents (e.g., antibodies) in the reaction chamber of an ACS by microfluidics allows the screening of multiple analytes in parallel. ${ }^{4}$ Though it presents less experimental details, a recent paper shows that Bayer and Boehringer Ingelheim are also active in this field. ${ }^{6} \mathrm{~A}$ device similar to an ACS but of a more simple design was furthermore recently applied for a C-reactive protein immunoassay. ${ }^{14}$

\section{Capillary flow in LOC systems other than for POC}

Apart from POC systems, capillary forces are also increasingly used for liquid propulsion in other LOC systems. Several typical LOC functionalities like juxtaposition of parallel flow streams, mixing and sample injection have recently been implemented by the use of capillary forces alone. Kim et al. demonstrated a flow system with two parallel streams driven by capillary forces (Fig. 3) ${ }^{15}$ The device uses the upstream flow control element shown in Fig. 1b, with an additional local tuning of the hydrophilicity at the merging area. By tailoring the hydrodynamic resistance of the inlet channels, the relative width of the parallel streams could be changed. The inclusion of a pressure balancer between reservoirs prevented backflow (Fig. 3). Chen et al. showed a microfluidic mixer driven by capillary pumping. Liquid is spontaneously imbibed between two glass plates, of which one has an asymmetric groove pattern, causing mixing. ${ }^{16}$

There is elegance in the simple singlestep capillary electrophoresis system demonstrated by Ono and Fujii, which relies on capillary force for sample and polymer solution loading into a device for DNA separation. ${ }^{17}$ Fig. 4 shows 


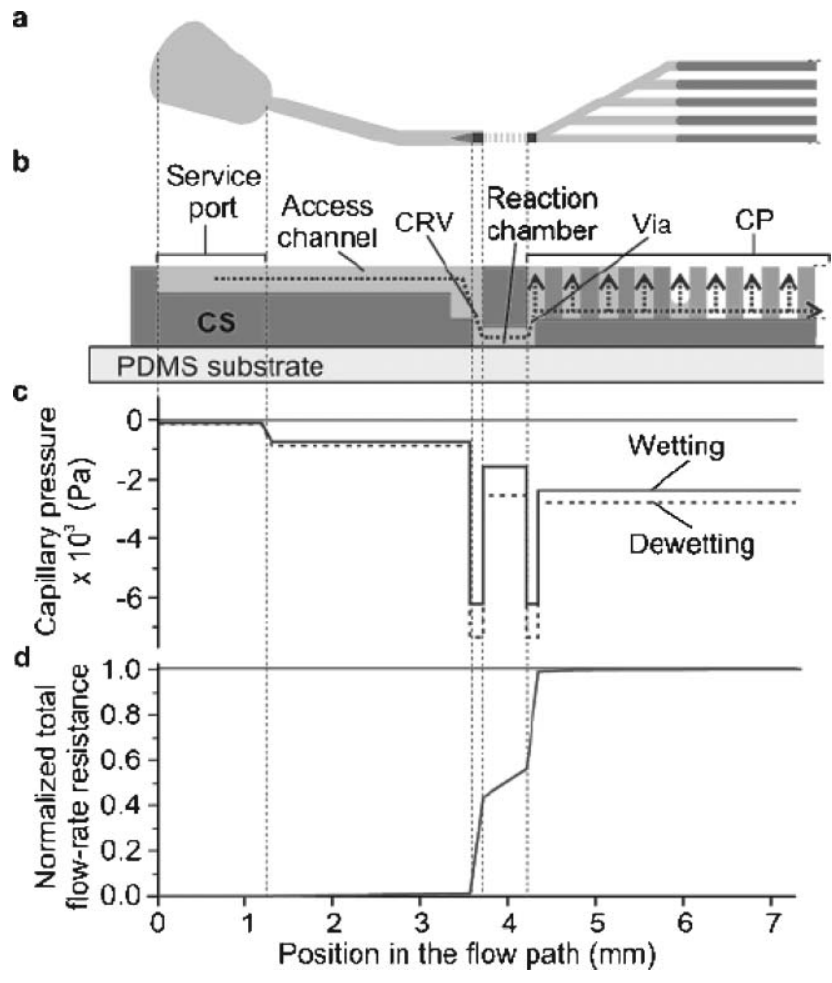

Fig. 2 (a) Schematic of a capillary system (CS) viewed from above. (b) The flow of liquid (arrows) is superposed to the cross section (not to scale) of the CS. (c) The capillary pressure at the front of water is calculated in all points of the CS for a filling (advancing; solid line) and an emptying (receding; dashed line) liquid front. The liquid flows toward a zone of the CS having a lower capillary pressure (more negative value), and once filled, the reaction chamber is protected from drying by a capillary retention valve (CRV). (d) The flow resistance of the CS is nearly invariant once the liquid has passed the reaction chamber, enabling the transport of multiple samples of different solutions under similar flow conditions. Reprinted from ref. 3 with permission from the publisher.

how the use of stop channels (upstream flow control) and air vents enables the introduction by capillary forces of both sample and polymer solution from sample introduction ports into the

channel system without the formation of air bubbles. In a next step the electrical field is applied to create separation. A very simple electrical setup can thus be used with just two electrodes.

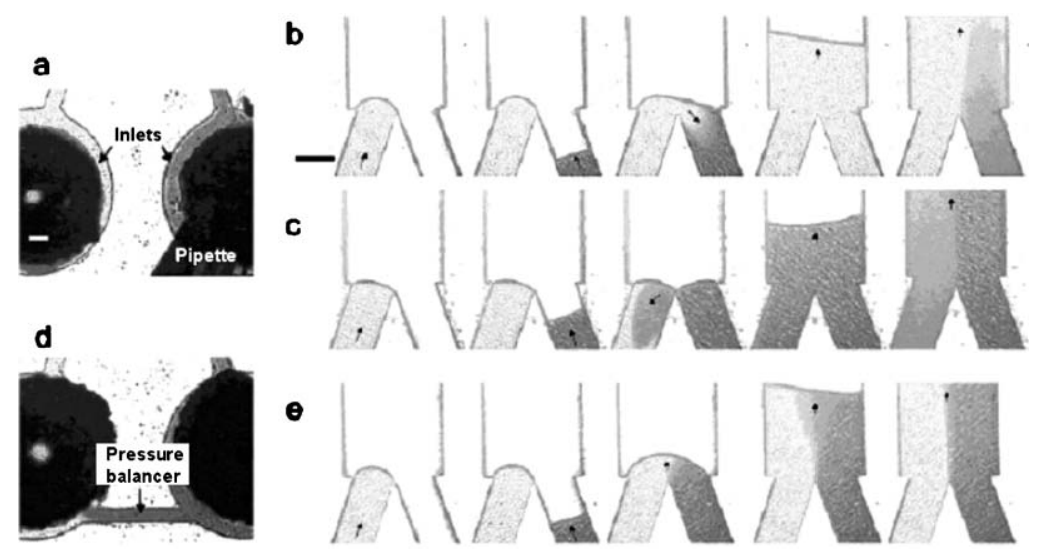

Fig. 3 Dependence of flow patterns on the existence of pressure balancer (width $140 \mu \mathrm{m}$ ). Here, $w / h=2.5$ and $h=80 \mu \mathrm{m}$. Scale bars, $200 \mu \mathrm{m}$. (a-c) Without a pressure balancer, reentrant flows occur, resulting in disturbances of volumetric flow rates ratio in each stream. (d, e) With a pressure balancer, the merged two streams pass through the junction equally, resulting in a parallel laminar flow. Reprinted from ref. 15 with permission from the publisher.
Other recent examples of the use of capillarity in LOC systems are the work of Stiles et al., who reports on a hydrodynamic focusing system that can be operated both by capillary force and vacuum, ${ }^{18}$ and of Vestad et al. who combines pumping by capillary pressure with valving by local depression of PDMS in microfluidic devices for diagnostic applications. ${ }^{19}$ A number of instances can furthermore be given where capillarity is used in the sample-preparation phase. Wallmann et al. demonstrated a microsystem for protein analysis by MALDI-MS that uses capillary pressure for sample transport. ${ }^{20}$ Capillarity-driven flow between micromachined ridges on an open surface has been used to prepare sample for X-ray fluorescence. $^{21}$ The ridges assisted in an equal distribution of the sample. Capillary flow between two hydrophobic plates where one of the plates is patterned with a hydrophilic pattern was demonstrated by Bouaidat et al. and applied to patterning of cells and proteins. ${ }^{22}$ An interesting property of this flow is that the flow path, by pinning on two sides is bordered by gas, reminiscent of the innovative work of Zhao et al..$^{23}$

Capillarity-regulated microfluidics in hydrophobic networks was demonstrated by the group of Ahn, who employed devices of hydrophobic plastics $\left(\theta>90^{\circ}\right.$ in eqn (1)). ${ }^{8}$ Liquid control on these devices is performed by local channel constrictions. Because propulsion is not automatic in hydrophobic channels, prepressurized air pockets were employed that were actuated by heat-induced membrane puncture. ${ }^{24}$

\section{Capillarity for surface treatment or self assembly}

A separate and exciting category of devices that uses capillarity is that of the dip-pen, and the microfluidic probe ${ }^{4}$ or microfluidic fountain pen. ${ }^{25}$ With such devices capillary forces pull liquid from a tip surface or a capillary lumen onto a surface via a capillary bridge, making them promising for surface treatment or, if the liquid can again be removed by a second capillary for example by an ACS, for surface analysis. ${ }^{26}$ Capillary forces are furthermore increasingly used for manufacturing purposes or for (self) assembly, for example of colloidal 


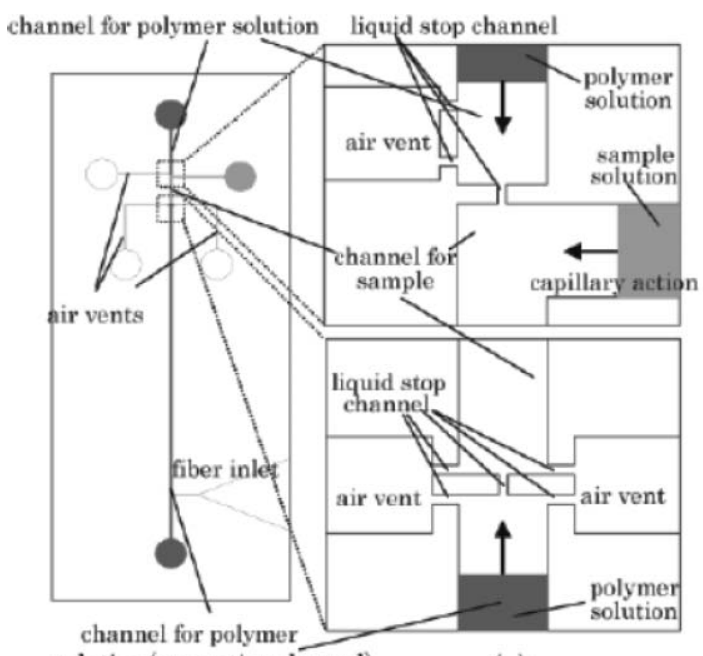

solution (separation channel)

(a)

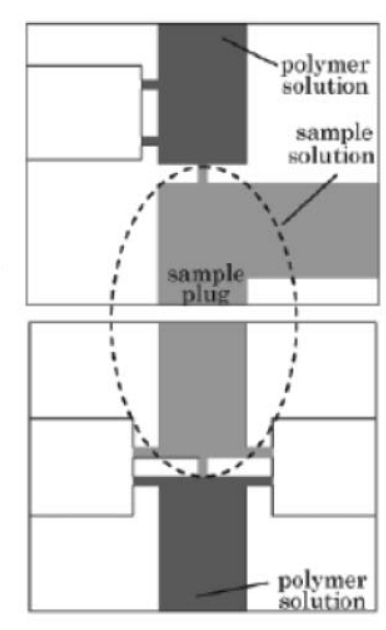

(b)

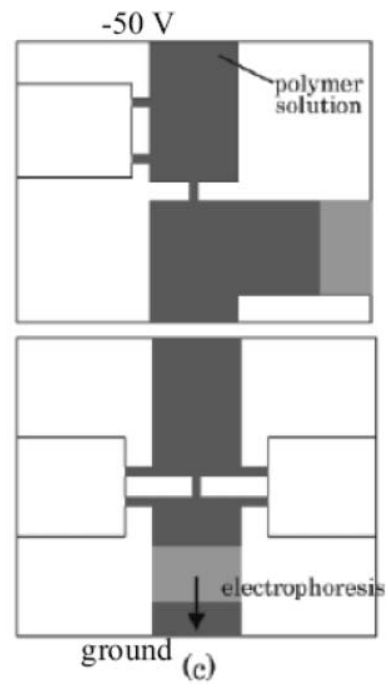

ground (c)

Fig. 4 Design of a PDMS device and plug formation scheme for single-step CE. (a) Introduction of reagents by capillary action. (b) Stop at liquid stop channels. (c) Plug injection for electrophoresis. Reprinted from ref. 17 with permission.

aggregates or proteins ${ }^{27}$ or to dock cells at specified positions on a microfluidic channel surface prior to assay. ${ }^{28}$

\section{Conclusion}

Capillarity-driven flow can with good success be used in LOC systems, especially because it scales well. The resulting autonomous or 'automatic' behaviour is of great use in POC devices, because it obviates the need for additional pumping or actuation equipment. Local changes of the channel geometries allow for complicated flow patterns and flow timing to be programmed, again without the need for external control equipment. Because of these obvious advantages, it is to be expected that capillarity will gain greater importance for LOC applications, especially if innovative device designs are produced.

Jan C. T. Eijkel

BIOS/Lab-on-a-Chip Group, MESA+

Research Institute, University of

Twente, The Netherlands

j.c.t.eijkel@utwente.nl

Albert van den Berg

BIOS/Lab-on-a-Chip Group, MESA+

Research Institute, University of

Twente, The Netherlands

\section{References}

1 D. D. Cunningham, Anal. Chim. Acta, 2001, 429, 1.
2 P. F. Man, C. H. Mastrangelo, M. A. Burns and D. T. Burke, in Proceedings of the IEEE Micro-Electro-Mechanical Systems Meeting, 25th-29th January 1998, Heidelberg, Germany, IEEE Press, Piscataway, 1998, p. 45.

3 D. Juncker, H. Schmid, U. Drechsler, H. Wolf, M. Wolf, B. Michel, N. de Rooij and E. Delamarche, Anal. Chem., 2002, 74, 6139.

4 E. Delamarche, D. Juncker and H. Schmid, Adv. Mater., 2005, 17, 2911.

5 D. C. Duffy, H. L. Gillis, J. Lin, N. F. Sheppard, Jr. and G. J. Kellogg, Anal. Chem., 1999, 71, 4669.

6 M. J. Pugia, G. Blankenstein, R. P. Peters, J. A. Profitt, K. Kadel, T. Willms, R. Sommer, H. H. Kuo and L. S. Schulman, Clin. Chem., 2005, 51, 1923.

7 M. R. McNeely, M. K. Spute, N. A. Tusneem and A. R. Oliphant, in Proceedings of the International SPIE Conference, Santa Clara, 1999, The International Society of Optical Engineering, Bellingham, WA, USA, 1999, vol. 3877 , pp. $210-220$.

8 C. H. Ahn, J. W. Choi, G. Beaucage, J. H. Nevin, J. B. Lee, A. Puntambekar and J. Y. Lee, Proc. IEEE, 2004, 92, 154.

9 J. Melin, N. Roxhed, G. Gimenez, P. Griss, W. van der Wijngaart and G. Stemme, Sens. Actuators, B, 2004, 100, 463.

10 S. Wilson and S. Howell, Biochem. Soc Trans., 2002, 30, 794.

11 S. Qian and H. H. Bau, Anal. Biochem., 2003, 322, 89.

$12 \mathrm{H}$. van Damme, T. Van Velthoven, E. Kaelen and E. Pelssers, Clin. Chem., 1997, 43, 369.

13 S. Cesaro-Tadic, G. Dernick, D. Juncker, G. Buurman, H. Kropshofer, B. Michel, C. Fattinger and E. Delamarche, Lab Chip, 2004, 4, 563.

14 K. Hosokawa, M. Omata, K. Sato and M. Maeda, Lab Chip, 2006, 6, 236.
15 S.-J. Kim, Y. T. Lim, H. Yang, Y. B. Shin, K. Kim, D.-S. Lee, S. H. Park and Y. T. Kim, Anal. Chem., 2005, 77, 6494.

16 C. F. Chen, C. F. Kung, H. C. Chen, C. C. Chu, C. C. Chang and F. G. Tseng, J. Micromech. Microeng., 2006, 16, 1358.

$17 \mathrm{~K}$. Ono and T. Fujii, in Micro Total Analysis Systems '05, ed. K. F. Jensen, J. Han, D. J. Harrison and J. Voldman, TRF, San Diego, CA, USA, 2005, p. 271.

18 T. Stiles, R. Fallon, T. Vestad, J. Oakey, D. W. M. Marr, J. Squier and R. Jimenez, Microfluid. Nanofluid., 2005, 1, 280.

19 T. Vestad, D. W. M. Marr and J. Oakey, J. Micromech. Microeng., 2004, 14, 1503.

20 L. Wallman, S. Ekström, G. MarkoVarga, T. Laurell and J. Nilsson, Electrophoresis, 2004, 25, 3779.

21 K. Tsuji, T. Emoto, Y. Nishida, K. Tsutsumimoto, K. Nakano, E. Tamaki, Y. Kikutani, A. Hibara and T. Kitamori, in Micro Total Analysis Systems '05, ed. K. F. Jensen, J. Han, D. J. Harrison and J. Voldman, TRF, San Diego, CA, USA, 2005, p. 991.

22 S. Bouaidat, O. Hansen, H. Bruus, C. Berendsen, N. K. Bau-Madsen, P. Thomsen, A. Wolff and J. Jonsmann, Lab Chip, 2005, 5, 827.

23 B. Zhao, J. S. Moore and D. J. Beebe, Science, 2001, 291, 1023.

24 A. Puntambekar, H. J. Cho, C.-C. Hong, J.-W. Choi, C. H. Ahn, S. Kim and V. B. Makhijani, Lab Chip, 2002, 2, 213.

25 S. Deladi, N. R. Tas, J. W. Berenschot, G. J. M. Krijnen, M. J. de Boer, J. H. de Boer, M. Peter and M. C. Elwenspoek, Appl. Phys. Lett., 2004, 85, 5361.

26 D. Juncker, H. Schmid and E. Delamarche, Nat. Mater., 2005, 4, 622.

27 K. Nagayama, Colloids Surf., 1996, 109, 363.

28 M. C. Park, J. Y. Hur, K. W. Kwon, S.-H. Park and K. Y. Suh, Lab Chip, 2006, 6, 988 . 